\title{
Karyotype variation is indicative of subgenomic and ecotypic differentiation in switchgrass
}

\author{
Hugh A Young ${ }^{1}$, Gautam Sarath² and Christian M Tobias ${ }^{1 *}$
}

\begin{abstract}
Background: Karyotypes can provide information about taxonomic relationships, genetic aberrations, and the evolutionary origins of species. However, differentiation of the tiny chromosomes of switchgrass (Panicum virgatum L.) and creation of a standard karyotype for this bioenergy crop has not been accomplished due to lack of distinguishing features and polyploidy.

Results: A cytogenetic study was conducted on a dihaploid individual $(2 n=2 X=18)$ of switchgrass to establish a chromosome karyotype. Size differences, condensation patterns, and arm-length ratios were used as identifying features and fluorescence in-situ hybridization (FISH) assigned 5S and 45S rDNA loci to chromosomes 7 and 2 respectively. Both a maize CentC and a native switchgrass centromeric repeat (PviCentC) that shared $73 \%$ sequence identity demonstrated a strong signal on chromosome 3. However, only the PviCentC probe labeled the centromeres of all chromosomes. Unexpected PviCentC and 5S rDNA hybidization patterns were consistent with severe reduction or total deletion of these repeats in one subgenome. These patterns were maintained in tetraploid and octoploid individuals. The $455 \mathrm{rDNA}$ repeat produced the expected number of loci in dihaploid, tetraploid and octoploid individuals. Differences observed at the 5S rDNA loci between the upland and lowland ecotypes of switchgrass provided a basis for distinguishing these subpopulations.

Conclusion: Collectively, these results provide a quantitative karyotype of switchgrass chromosomes. FISH analyses indicate genetic divergence between subgenomes and allow for the classification of switchgrass plants belonging to divergent genetic pools. Furthermore, the karyotype structure and cytogenetic analysis of switchgrass provides a framework for future genetic and genomic studies.

Keywords: Bioenergy, Switchgrass, Cytogenetics, CHIAS IV, Perennial biofeedstocks, Dihaploids, Renewable energy, Polyploidy
\end{abstract}

\section{Background}

Polyploidy, the heritable duplication of whole genomes, is a key feature of plant diversification and is found in most, if not all, plant taxa [1]. Polyploidization can lead to speciation or creation of distinct reproductivelyisolated cytotypes within a species. An ancient genomedoubling event and subsequent gene loss has shaped the genomes of all grass species [2]. Though an estimated $30-80 \%$ of angiosperms may be polyploid [3], the relative frequencies of allopolyploids which arise through hybridization of different species versus autopolyploids

\footnotetext{
* Correspondence: christian.tobias@ars.usda.gov

${ }^{1}$ Genomics and Gene Discovery Research Unit, USDA-Agricultural Research Service, Western Regional Research Center, 800 Buchanan Street, Albany, CA 94710, USA

Full list of author information is available at the end of the article
}

which arise within a single species are difficult to ascertain. Evolutionary processes that mask the origins of polyploid evolution such as introgression, deletion, concerted evolution, and mutation may create uncertainty. There is possible recurrent hybridization of genomes over time and interracial or close interspecific hybridization of genomes may produce similar outcomes. Incongruent tree-topologies from independent marker sets such as chloroplast and nuclear markers can suggest the presence of reticulate relationships, but these are not conclusive [4].

The interspecific relationships of taxa in the subgenus Panicum sensu stricto (s.s.) that comprises approximately $100 \mathrm{C} 4$ grass species, including Panicum virgatum L. (switchgrass), haven't yet been elucidated and likely represent a complex situation [5]. Because switchgrass

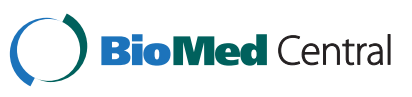


is being pursued as a promising feedstock for renewable energy production in the United States and elsewhere, these relationships are of importance for understanding the breadth of germplasm that might be useful in a breeding program. The plant's broad geographic range, water- and nutrient-use efficiency, and perennial growth habit make it ideal as a bioenergy crop [6-8]. The species encompasses multiple cytotypes with a basic chromosome number of nine $[9,10]$, and a range of ploidy levels from diploid $(2 \mathrm{x})$ to duodecaploid $(12 \mathrm{x})$ [11-14]. As a polyploid species, switchgrass exhibits two basic subgenomes that are genetically divergent but maintain complete or near complete disomic inheritance [15]. Two separate ecotypes are distinguished cytologically and geographically [16]. Lowland accessions dominate the southern portion of the species range and are mainly tetraploid, while the upland accessions are usually either tetraploid or octoploid and dominate northern latitudes [17]. Extensive aneuploidy has also been described, especially within populations of octoploids, but these are likely present in all populations at varying levels [18].

Although artificial crosses between switchgrass cytotypes have been largely unsuccessful [19,20], questions still remain regarding historical hybridization between cytotypes, between upland and lowland ecotypes, and between several closely related species in the Panicum subgenus. These species may represent a common gene pool that has undergone repeated hybridization during "secondary contacts" of once isolated populations [21]. A recent analysis of switchgrass collections has demonstrated that there are two distinct centers of genetic diversity for lowland accessions represented by the Southern Great Plains and Eastern Gulf Coast while upland accessions appear genetically as one broadly distributed tetraploid and two octoploid lineages [22]. Using chloroplast sequence polymorphisms, molecular clock estimates have indicated that lowland and upland accessions diverged as early as 1.3 million years ago, but have possibly diverged on several occasions during recent cycles of glaciation $[23,24]$.

In light of these uncertainties, independent methods to characterize genome structure would be useful for effective evaluation and utilization of germplasm resources. Cytogenetic analysis using in situ hybridization techniques have proven very useful in resolving genome constitution in polyploids and is an important tool in chromosome karyotyping [25]. In polyploid plants with small and highly similar chromosomes, karyotyping is aided by fluorescence in situ hybridization (FISH) using labeled total genomic DNA, repetitive sequences, or single copy probes. In particular, variation found at rDNA loci (45S and 5S rDNA) can sometimes be used to differentiate subgenomes or to distinguish between ecotypes of a species [26,27]. Chromosome reduction, breakage, or fusion during or after polyploidization can result in a gain or loss of these tandem repeat sequences. In the Triticeae, for example, both the location and order of rDNA loci differ extensively among related species [28]. FISH analyses using repetitive probes can further enable chromosome identification, and have been successfully employed in maize [29], rice [30], sugarcane [31], soybean [32], and pine [33].

In complex polyploid organisms such as switchgrass, the development of genotypes with reduced chromosome numbers would prove useful for breeding and genetic research [34]. Haploid plants, whether derived from a diploid or a polyploid, have half the chromosome number of the euploid form. Therefore, "haploid" plants derived from switchgrass tetraploids will have two copies of the basic chromosome number of nine $(2 n=2 X=18)$. With a true haploid number of nine, the term "dihaploid" has been used to describe androgenic switchgrass lines containing two sets of homologous chromosomes [35] as well as gynogenic lines containing two sets of non-pairing homoeologous chromosomes [36]. The "dihaploid" terminology as well as the utility of such plant lines has been previously described for potato, barley, wheat, and several other species [37-39].

Early cytological studies in switchgrass focused mainly on overall counts of chromosomes rather than their individual morphology and molecular structure [40]. Our purpose here is to describe a quantitative karyotype of $P$. virgatum $\mathrm{L}$. To simplify analysis, we have used a dihaploid line $(2 n=2 X=18)$ derived from a lowland tetraploid individual through gynogenesis [36]. The term "dihaploid" is used to indicate the polyploid origin of the line and the homoeologous constitution of its subgenomes. Here we establish a standard reference karyotype that identifies visible features of individual switchgrass chromosomes and allows their unique discrimination. We demonstrate the presence of both additive and nonadditive loci associated with rDNA and centromeric repeats, indicating subgenome divergence. We also document karyotype differences between upland and lowland genotypes.

\section{Results}

\section{Chromosome measurement data}

Somatic chromosome counts made from root tip squash preparations confirmed the presence of $2 n=36$ metaphase chromosomes in the tetraploid cultivar Kanlow (Figure 1a), which agrees with published data [14,41]. Chromosome numbers for ALB280 were shown to be $2 n=18$ based on data from more than 50 metaphase plate preparations (Figure 1b). Image analyses of metaphase spreads from ALB280 (Figure 1b) were performed to produce the switchgrass karyotype. Digital measurements 


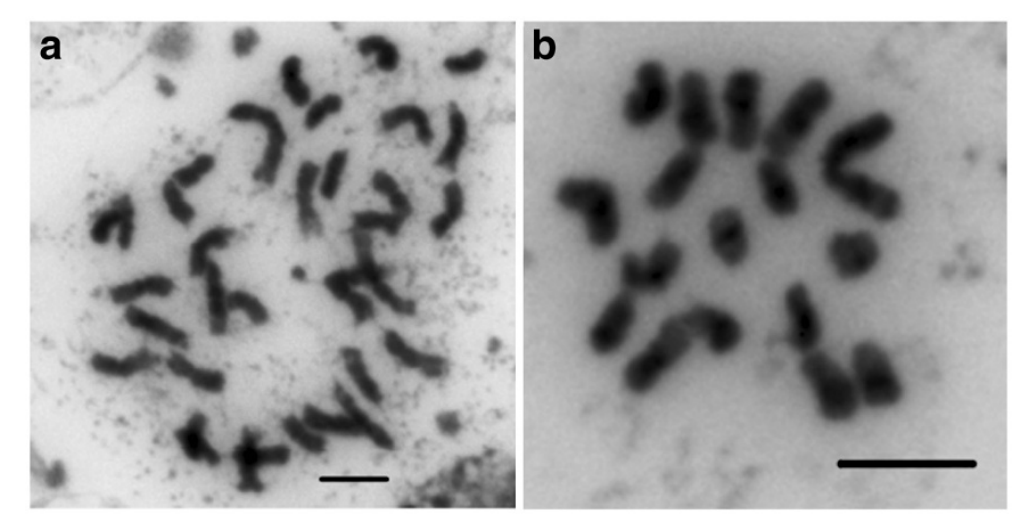

Figure 1 Genome reduction in dihaploid individuals. Chromosome squashes prepared from root tip cells confirmed $2 n=36$ chromosomes in the tetraploid cultivar Kanlow (a) and $2 n=18$ in dihaploid ALB280 (b). Scale bars $=5 \mu \mathrm{m}$.
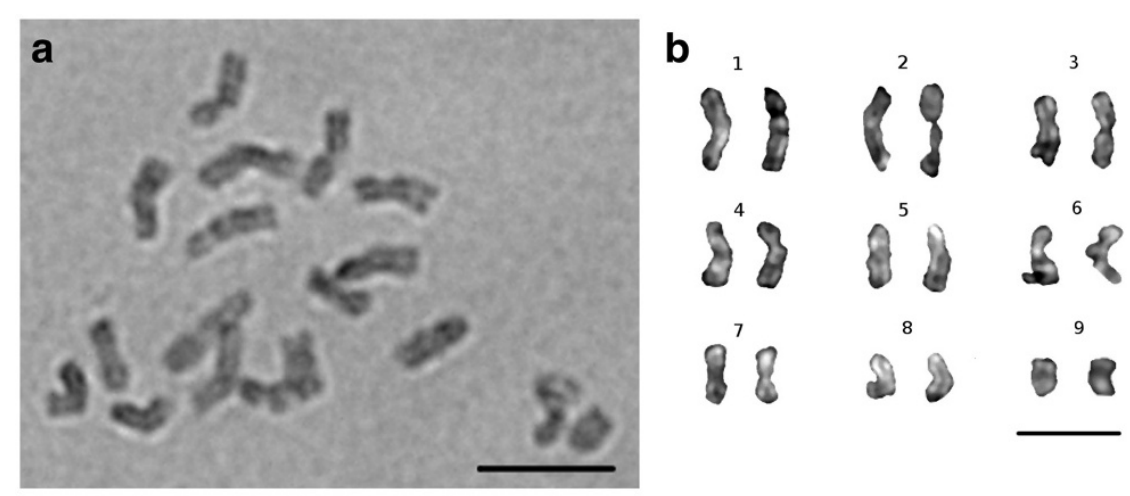

C

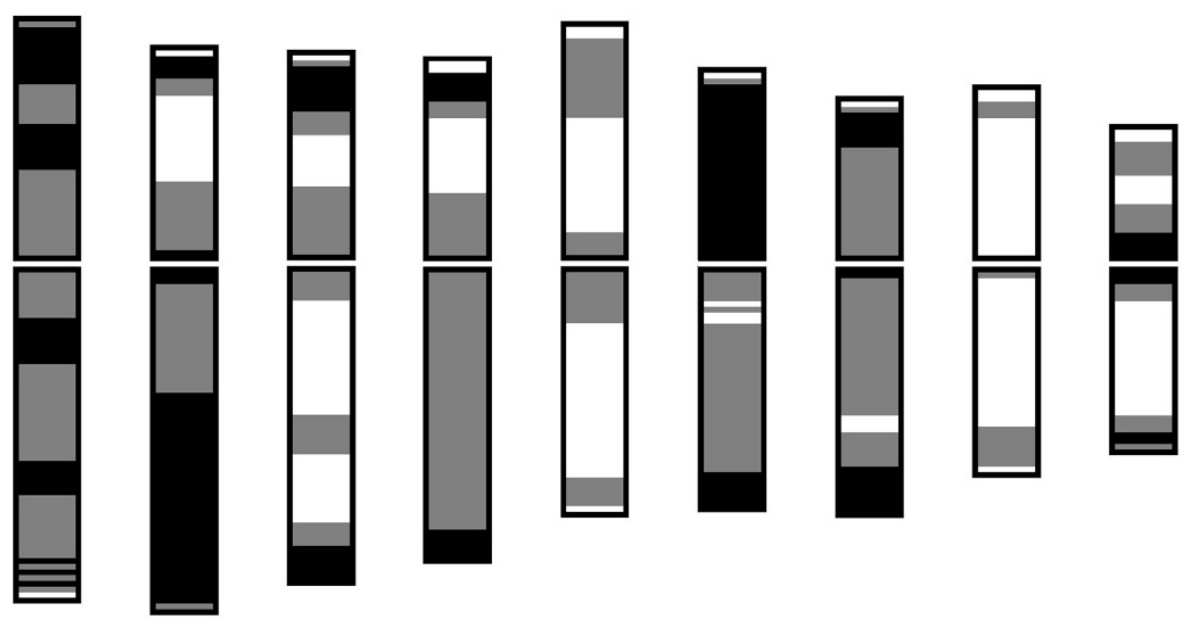

Figure 2 Switchgrass Karyogram and Condensation Pattern (CP). Mitotic prometaphase chromosome spreads were stained with

acetocarmine, resulting in uneven condensation patterns (a). Homoeologous chromosomes were paired based on length, arm ratio, and CP data to develop a karyogram. The basic chromosome number of $n=9$ is indicated (b). CP measurements were averaged for each chromosome across 10 mitotic spread images, resulting in a quantitative ideogram (c). Dark regions indicate highly-condensed heterochromatic areas, gray indicates intermediate condensation, and white indicates euchromatic areas. Scale bars $=5 \mu \mathrm{m}$. 


\begin{tabular}{|c|c|c|c|}
\hline Chromosome & Length ${ }^{\mathrm{a}}(\mu \mathrm{m} \pm \mathrm{SE})$ & Arm Ratio $^{\mathrm{b}}(r \pm \mathrm{SE})$ & ) Centricity Class \\
\hline 1 & $4.10 \pm 0.25$ & $1.30 \pm 0.09$ & $\mathrm{~m}$ \\
\hline 2 & $3.73 \pm 0.30$ & $1.31 \pm 0.06$ & $\mathrm{~m}$ \\
\hline 3 & $3.38 \pm 0.18$ & $1.15 \pm 0.07$ & $\mathrm{~m}$ \\
\hline 4 & $3.26 \pm 0.16$ & $1.28 \pm 0.13$ & $\mathrm{~m}$ \\
\hline 5 & $3.05 \pm 0.18$ & $1.22 \pm 0.16$ & $\mathrm{~m}$ \\
\hline 6 & $2.82 \pm 0.16$ & $1.21 \pm 0.06$ & $\mathrm{~m}$ \\
\hline 7 & $2.61 \pm 0.17$ & $1.30 \pm 0.08$ & $\mathrm{~m}$ \\
\hline 8 & $2.35 \pm 0.11$ & $1.18 \pm 0.06$ & $\mathrm{~m}$ \\
\hline 9 & $2.05 \pm 0.16$ & $1.21 \pm 0.04$ & $\mathrm{~m}$ \\
\hline
\end{tabular}

${ }^{a}$ Average value of 10 chromosome pairs $(\mu \mathrm{m})$.

${ }^{\mathrm{b}}$ Arm ratio $\mathrm{r}$ (long/short) according to Levan et al. [42].

$S E=$ standard error; $m=$ metacentric.

allowed for homoeologous chromosomes to be paired together and the base number $n=9$ to be presented in a karyogram, from longest to shortest (Figure 2a,b). Morphological data from ten complete and undistorted chromosome spreads are presented in Table 1, indicating that switchgrass chromosomes are small to medium in length, ranging from $2.05 \pm 0.16 \mu \mathrm{m}$ to $4.10 \pm 0.25 \mu \mathrm{m}$. Identification of the centromeres via FISH analyses (data presented below) allowed for measurement of chromosome arms and calculation of arm ratios $(r)$. All chromosomes were classified as metacentric (m) based on parameters described by Levan et al. [42].

\section{Condensation pattern}

Chromosomes exhibit uneven staining due to variation in condensation pattern $(\mathrm{CP})$ along their length. This distinctive $\mathrm{CP}$, especially at the prometaphase stage of mitosis, was utilized as a diagnostic measurement for chromosome identification. CHIAS IV software [43] takes advantage of this uneven staining pattern and was used to generate a distinctive $\mathrm{CP}$ profile for each of the nine switchgrass chromosomes (Figure 2c). The same ten chromosome spread images used to generate the morphological data in Table 1 were analyzed with CHIAS IV software. Homoeologous chromosomes were paired in each prometaphase spread and then averaged together with homologs across all ten spreads. The resulting ideogram based on chromosome length and CP is presented in Figure 2c. Gray scale images allowed for distinct characterization of each switchgrass chromosome based on features such as the large, condensed region on the long arm of Chromosome 2, interstitial constrictions on both arms of Chromosome 1, and the large white areas indicating euchromatic regions of Chromosomes 5 and 8 (Figure 2c). Taken together with the length and arm ratio data, CP profiles allow each chromosome to be distinguished from all others in the karyotype.

\section{Localization of centromeres}

A plasmid probe developed from the maize centromere repeat sequence CentC [44] (GenBank AF078922.1) was used for FISH of switchgrass chromosomes. Only one CentC signal was observed in mitotic chromosome spreads of dihaploid ALB280 $(2 n=2 X=18)$ (Figure 3a). Upon FISH analysis of the lowland tetraploid cultivar Alamo $(2 n=4 X=36)$, two CentC signals were evident (Figure 3b). Moreover, four CentC signals were present in chromosome spreads of the upland octoploid cultivar Grenville $(2 n=8 X=72)$ (Figure 3c). Chromosome length and arm ratio data of ALB280 were used to determine that the CentC probe hybridizes to Chromosome 3 of the basic karyotype (Figure 4).

BLAST analysis of switchgrass 454 sequence data using the maize CentC and rice CentO sequences resulted in a subset of potential centromere-specific repeat sequences found in the switchgrass genome that, on average, were $73 \%$ identical to maize CentC (AF078922.1). Primers designed from the corresponding consensus sequence were used to amplify and label a switchgrass-specific centromere repeat sequence for FISH analysis. The labeled switchgrass centromere probe (PviCentC) produced a fluorescent signal on all 18 chromosomes of ALB280 (Figure 3d). In addition, one chromosome of ALB280 demonstrated a centromeric FISH signal significantly brighter than all the others. This PviCentC signal pattern was also present in all tetraploid cytotypes tested, where all centromeres were labeled and two chromosomes demonstrated a very high level of fluorescence (Figure 3e, cv. Kanlow). Octoploid cytotypes maintained this pattern, demonstrating four strong FISH signals on specific chromosomes while all others contained equal intensity signals at their centromeres (Figure 3f, cv. Cave-in-Rock). Length, arm ratio, and condensation pattern analyses identified the high fluorescing chromosomes as Chromosome 3, which is consistent with the maize CentC probe from which PviCentC was derived.

\section{S rDNA localization}

The distribution of $45 \mathrm{~S}$ rDNA on mitotic metaphase chromosomes of switchgrass was detected with FISH using the wheat pTa71 plasmid probe [45]. These results demonstrate that one pair of $45 \mathrm{~S}$ rDNA signal was detected in chromosome spreads of ALB280, suggesting that the basic karyotype $(n=9)$ has one chromosome containing the $45 \mathrm{~S}$ rDNA sequence (Figure $3 \mathrm{~g}$ ). When the tetraploid, lowland individual AP13 is probed, two pairs of $45 \mathrm{~S}$ rDNA signals are present (Figure $3 \mathrm{~h}$ ). This pattern of one $45 \mathrm{~S}$ rDNA signal per base genome $(n=9)$ also persisted in an individual from the octoploid upland cultivar Caddo, where eight $45 \mathrm{~S}$ rDNA signals are visible (Figure 3i). Analysis of the morphology and FISH staining patterns in multiple spreads indicates 

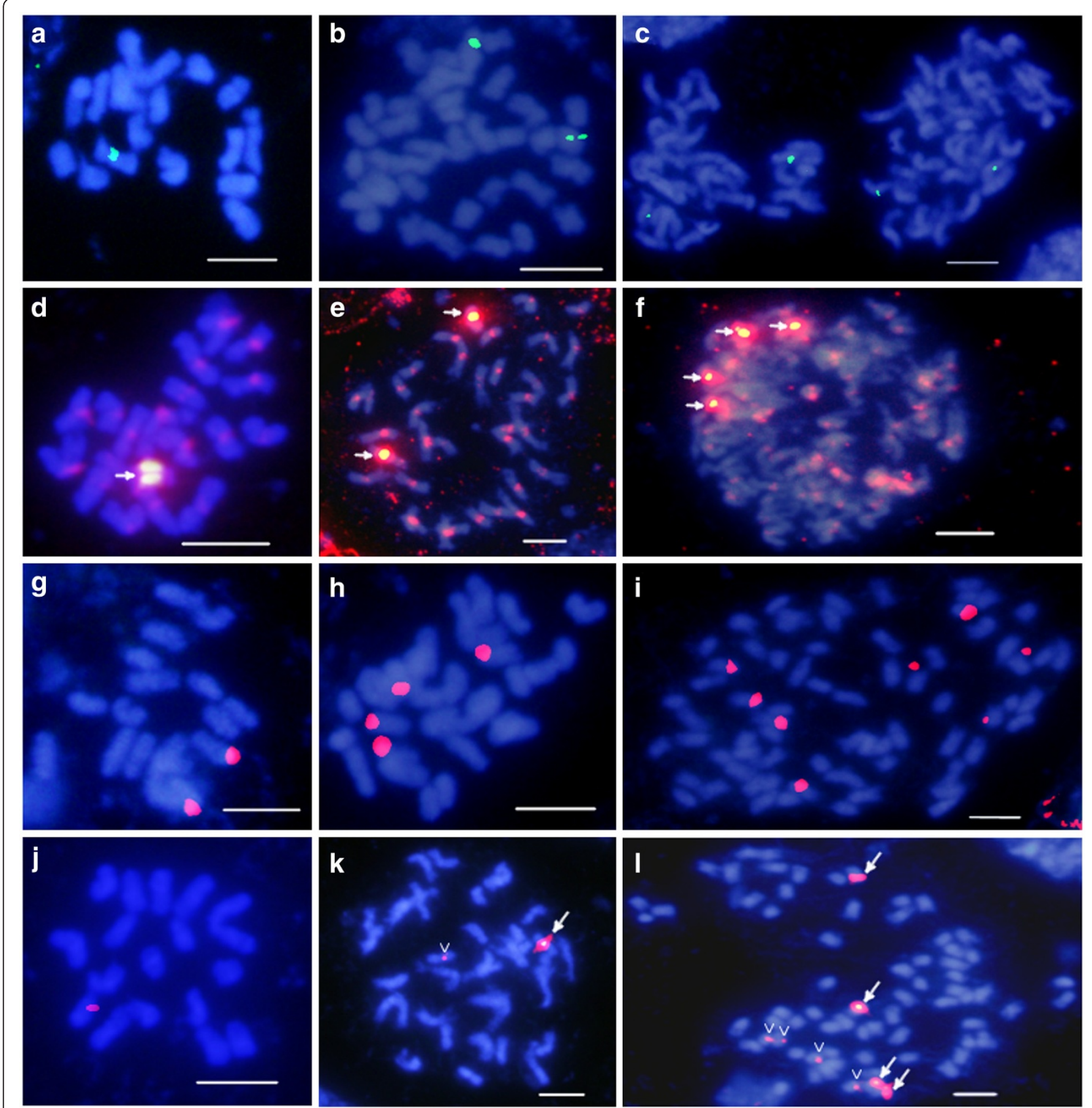

Figure 3 FISH analyses of switchgrass chromosomes. Comparative FISH analysis of switchgrass chromosomes was conducted using four different probe sequences: CentC (a,b,c); PviCentC (d,e, $\mathbf{f}) ; 45 S$ rDNA ( $\mathbf{g}, \mathbf{h}, \mathbf{i}) ; 5 S$ rDNA $(\mathbf{j}, \mathbf{k}, \mathbf{l})$. Three different switchgrass cytotypes were analyzed for each FISH probe: dihaploid $2 n=2 X=18(\mathbf{a}, \mathbf{d}, \mathbf{g}, \mathbf{j})$; tetraploid $2 n=4 X=36(\mathbf{b}, \mathbf{e}, \mathbf{h}, \mathbf{k})$; octoploid $2 n=8 X=72(\mathbf{c}, \mathbf{f}, \mathbf{i}, \mathbf{l})$. CentC signals $(\mathbf{a}, \mathbf{b}, \mathbf{c})$ are indicated in green, whereas all other probes are labeled with red fluorescence. White arrows in $\mathbf{d}, \mathbf{e}$, and $\mathbf{f}$ indicate the strong PviCentC signal present on Chromosome 3 of the basic karyotype $(n=9)$. White arrows in $\mathbf{k}$ and $\mathbf{I}$ indicate "strong" 5 S rDNA signals as compared to the "weak" 55 rDNA signals in each image (open arrowheads). Scale bars $=5 \mu \mathrm{m}$.

that the 45S rDNA signal is found near the terminal end of the short arm of Chromosome 2 (Figure 4).

\section{S rDNA localization}

Sequence data from 454 reads of dihaploid switchgrass ALB280 were used to BLAST against 5S rDNA sequences of several grass species, including sorghum, rice, maize, and wheat. The resulting sequence hits were used to amplify and fluorescently label a 314-basepair orthologous $5 \mathrm{~S}$ rDNA sequence in switchgrass. These $5 \mathrm{~S}$ rDNA FISH probes hybridized to a single chromosome of dihaploid ALB280 and to two chromosomes of the 

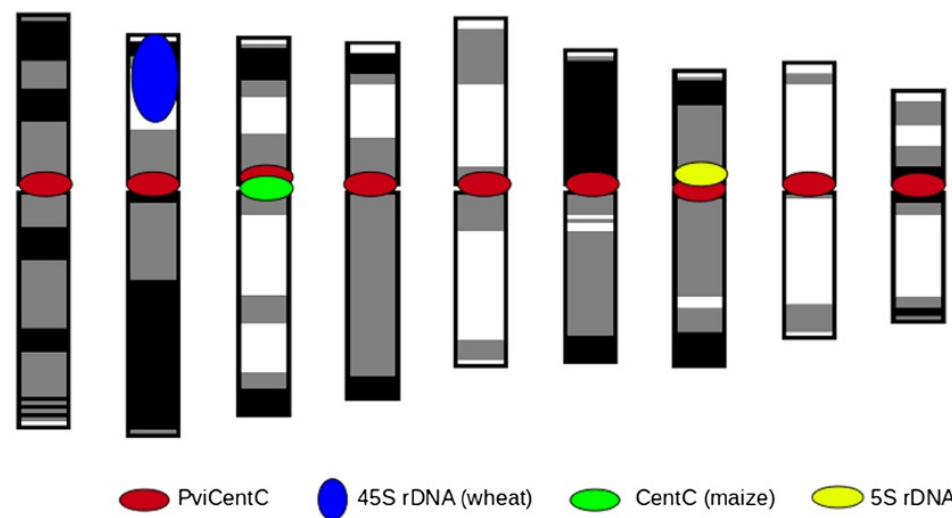

$45 \mathrm{~S}$ rDNA (wheat)

CentC (maize)

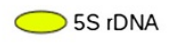

\begin{tabular}{c|c|c|c|c|c|c|c|c|c}
\hline Chromosome & $\mathbf{1}$ & $\mathbf{2}$ & $\mathbf{3}$ & $\mathbf{4}$ & $\mathbf{5}$ & $\mathbf{6}$ & $\mathbf{7}$ & $\mathbf{8}$ & $\mathbf{9}$ \\
\hline Length $^{\mathrm{a}}$ & $4.10 \pm$ & $3.73 \pm$ & $3.38 \pm$ & $3.26 \pm$ & $3.05 \pm$ & $2.82 \pm$ & $2.61 \pm$ & $2.35 \pm$ & $2.05 \pm$ \\
( $\boldsymbol{\mu m} \pm \mathrm{SE})^{0.25}$ & 0.30 & 0.18 & 0.16 & 0.18 & 0.16 & 0.17 & 0.11 & 0.16 \\
\hline Arm Ratio $^{\mathrm{b}}$ & $1.30 \pm$ & $1.31 \pm$ & $1.15 \pm$ & $1.28 \pm$ & $1.22 \pm$ & $1.21 \pm$ & $1.30 \pm$ & $1.18 \pm$ & $1.21 \pm$ \\
$(\mathrm{r} \pm \mathrm{SE})$ & 0.09 & 0.06 & 0.07 & 0.13 & 0.16 & 0.06 & 0.08 & 0.06 & 0.04 \\
\hline
\end{tabular}

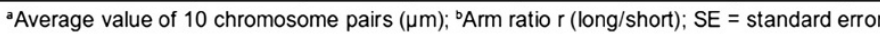

Figure 4 Quantitative karyotype of switchgrass chromosomes. An ideogram of the base $n=9$ switchgrass chromosomes is displayed with corresponding FISH signal probes. Length and arm ratio measurements are averaged across 10 chromosome pairs.

lowland tetraploid cultivar Kanlow (Figure 3j \& k, respectively). Quantitative measurement data of ALB280 chromosomes localize the 5S rDNA signal to an interstitial region on Chromosome 7 (Figure 4). In the FISH image of Kanlow chromosomes, one 5S rDNA signal is strong and one 5S rDNA signal is weak (Figure 3k). In upland octoploid ecotypes, FISH signals for 5S rDNA demonstrate a different pattern than what would be predicted based on dihaploid and tetraploid data. Instead of two strong and two weak signals, the upland octoploid cultivar Caddo shows four strong and four weak hybridization sites (Figure 3l). This suggests a unique difference between upland and lowland ecotypes at the $5 \mathrm{~S}$ rDNA locus.

\section{Ecotype variation at the $5 \mathrm{~S}$ rDNA locus}

To further examine differences between ecotypes of switchgrass at the $5 \mathrm{~S}$ rDNA locus, upland tetraploid cultivars Dacotah and Summer were analyzed via FISH and compared to the lowland tetraploids Kanlow and Alamo. Two strong 5S rDNA signals and two weak signals (Figure 5) were observed in the upland tetraploids, which is consistent with the pattern seen in upland octoploids of four strong and four weak $5 \mathrm{~S}$ rDNA signals (Figure 3l). In contrast, lowland tetraploids Kanlow and Alamo contain only one strong and one weak 5S rDNA signal (Figure 5). FISH data also support the conclusion that the dihaploid ALB280 has maintained one of the $5 \mathrm{~S}$ rDNA loci from its tetraploid progenitor [36].

\section{Discussion}

An accurate karyotype can incorporate physical measurements like total length and arm length ratios, but can also include landmarks such as heterochromatic knobs [46], patterns of chromatin condensation [43], and molecular features visualized by FISH [47]. Chromosome identification is critical for cytological analyses, as well as subsequent studies in genomics, taxonomy, and the evolution of polyploidy, enabling an understanding of the relationship between visible landmarks and genetic or physical map features [48]. To that end, the construction of a basic karyotype for switchgrass promises to facilitate genomic analyses. The somatic metaphase chromosomes of switchgrass are small, which may have limited examination of cytological features in earlier studies $[11,13,49]$. With the use of sophisticated imaging and molecular techniques, we are now able to present the first comprehensive karyotype for switchgrass that quantitatively distinguishes each of the nine base chromosomes of this bioenergy crop.

Use of a dihaploid line of switchgrass (ALB280) significantly simplified the karyotyping process. Acetocarmineand DAPI-stained chromosome spreads allowed for visual pairing of homoeologous chromosomes in ALB280 and produced a karyotype based on total and relative lengths as well as arm ratios. In our experiments, a single switchgrass root tip preparation yielded an average of 20 or more dividing cells (prophase to metaphase). Chromosome spreads often resulted in a high frequency of nuclei at the pro-metaphase stage of mitosis. Pro-metaphase 

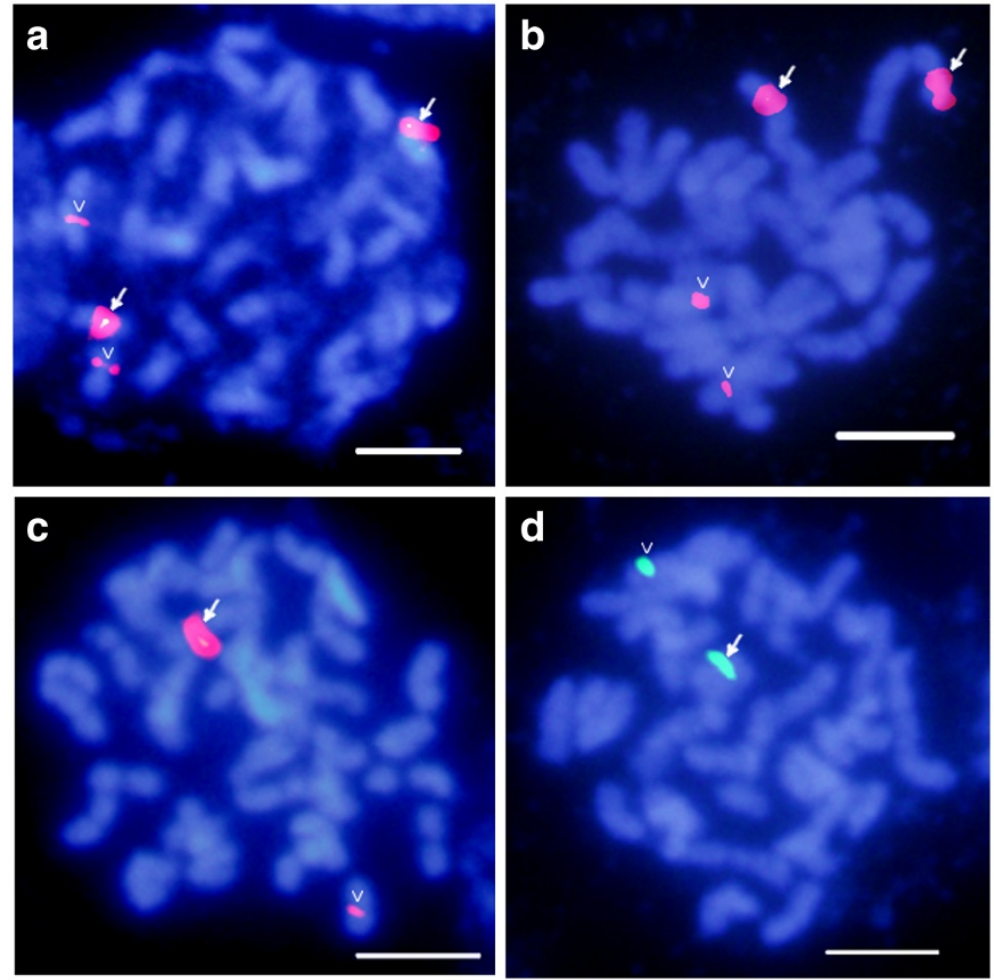

Figure 5 Ecotype variation at the 5S rDNA locus. FISH analyses of the 5S rDNA probe indicated signal variation between upland and lowland ecotypes. The upland tetraploid cultivars Dacotah (a) and Summer (b) were compared to the lowland tetraploid cultivars Kanlow (c) and Alamo (d). All FISH signals are of $5 \mathrm{~S} \mathrm{rDNA}$, but both red and green fluorescence were used. White arrows in each image indicate "strong" signal patterns in comparison to "weak" signals in the same image (open arrowheads). The double "snake-eye" signal found in panel a simply indicates labeling of individual chromatids on the same chromosome. Scale bars $=5 \mu \mathrm{m}$.

chromosomes demonstrated a characteristic condensation pattern $(\mathrm{CP})$ along their length, corresponding to the compactness of the chromatids, which was used to create a quantitative idiogram [50]. This approach has also been useful in cytological analysis of Brassica species, sugarcane, and rice [51-53]. In conjunction with physical measurement data, CP data allowed us to unambiguously identify the small metacentric chromosomes of switchgrass.

Although morphological and $\mathrm{CP}$ data suggest a balanced karyotype in the dihaploid line ALB280 $(2 n=2 X=18)$, FISH data presented here indicate that the subgenomes have different repetitive DNA content at PviCentC and 5S rDNA loci. This finding is in general agreement with the highly differentiated genomes indicated by linkage map data in which tetraploid ecotypes demonstrate fewer than expected markers mapping across subgenomes, and complete or near complete disomic inheritance [15]. It also agrees with the observation of 18 non-pairing univalents at diakinesis of meiosis in the dihaploid line [36]. FISH signal data at these genetic loci may point toward allopolyploid evolution of the switchgrass genome. However, these data are also consistent with natural loss of gene content following a whole genome duplication within a single species (autopolyploidy). To gain a greater understanding of the origins of switchgrass polyploidy, further phylogenetic analyses of the Panicum (s.s.) subgenus and/or genomic in situ hybridization (GISH) techniques should be used [1,27,54-56].

Under a simple additive model, FISH signals for the $5 \mathrm{~S}$ rDNA locus and the CentC locus would be expected to be present in 2, 4 , and 8 copies in the dihaploid, tetraploid, and octoploid lines, respectively. Surprisingly, only one FISH signal for these loci was observed in the dihaploid ALB280. In addition, the switchgrass-specific centromere probe, PviCentC, hybridized to all chromosomes of both subgenomes, but demonstrated a stronger FISH signal on Chromosome 3 of a single subgenome (same locus as maize CentC probe). This discrepancy in signal strength, particularly for the universal centromere probe PviCentC, suggests that a greater copy number of this repeat is present in Chromosome 3 of one subgenome than in all other chromosomes. Alternatively, or in conjunction, homology to the PviCentC FISH probe sequence may be much higher in Chromosome 3 than in all other labeled centromeres. The variation we observe at rDNA and centromeric loci may also be 
a result of the allogamous habit and selfincompatibility of switchgrass [20]. In outbreeding species of the genus Secale, high levels of repeat DNA polymorphism between homologous chromosomes have been documented [57]. Other outbreeding species in the Lolium and Lolium-Festuca complex demonstrate variation at rDNA loci $[58,59]$, suggesting that hemizygosity in switchgrass may result from outcrossing. Also contributing to non-additive FISH signal data may be the high frequency of switchgrass aneuploids, particularly among octoploid cytotypes $[18,40]$, which can lead to large-scale genetic changes and parental genome imbalance.

In our analyses of $45 \mathrm{~S}$ rDNA loci, pairs of telomeric FISH signals demonstrated a regular, additive pattern up the ploidy series. Among tetraploid cultivars, our data are consistent with those of Costich et al. [18] in which all tetraploids analyzed (upland and lowland) demonstrated two pairs of telomeric $45 \mathrm{~S}$ rDNA signals. However, in octoploid cultivars, Costich et al. [18] describe a large amount of variation in size, number, and location of $45 \mathrm{~S}$ rDNA signals. The variation in $45 \mathrm{~S}$ rDNA signal intensity seen in our analysis of the octoploid cv. Caddo (see Figure 3i) may suggest rDNA repeat variation and/or differences in probe hybridization affinity. With only a single upland octoploid (cv. Caddo) analyzed with $45 \mathrm{~S}$ rDNA in this study, our results likely demonstrate one of many chromosomal constitutions for switchgrass octoploids. Overall, FISH analyses of tetraploid and octoploid individuals support elimination of rDNA and centromere sequences and demonstrates that patterns of subgenome differentiation are broadly maintained.

Our data also demonstrate unique ecotype differences at $5 \mathrm{~S}$ rDNA loci. Variations in FISH signal patterns between upland and lowland tetraploids (and between lowland tetraploids and upland octoploids) provide features that distinguish these taxonomic divisions within switchgrass. We hypothesize that these variations in rDNA loci are related to both the phenotypic and geographic distribution differences observed between switchgrass ecotypes. In Oryza species, polymorphisms in the number, the chromosomal location, and the repeat length of rDNA loci have revealed species-specific and subgenome-specific FISH patterns [60]. The authors suggest that specific inversions, rDNA amplification, and locus transposition may have occurred during the process of Oryza evolution. Such a scenario may also be true for the divergence of switchgrass upland and lowland ecotypes. Classified cultivars of switchgrass are barely removed from the wild, and rapidly evolving rDNA loci are likely still undergoing change. The additive pattern of $5 \mathrm{~S}$ rDNA loci seen between lowland tetraploids (2 signals) and upland tetraploids (4 signals) may be indicative of chromosomal rearrangements and rDNA changes that ultimately result in habitat adaptability differences between the two ecotypes. In addition, the conserved doubling pattern of $5 \mathrm{~S}$ rDNA loci from upland tetraploid to upland octoploid further supports the maintenance of ecotype divergence across different cytotypes.

The presence of gene flow between switchgrass ecotypes and/or cytotypes has ramifications on the development and use of specific gene pools for switchgrass improvement. Therefore, knowledge of chromosome architecture and ploidy relationships is critical for cultivar development. Cytogenetic data presented here can be used to classify switchgrass plants according to ecotype and ultimately aid in identifying and isolating regionally adapted cultivars [61,62]. Switchgrass improvement through trait identification and breeding for significant heterotic effects also warrants the maintenance of independent gene pools $[63,64]$. Recent analyses of several putative upland and lowland accessions of switchgrass have identified the presence of natural hybrids between ecotypes as well as evidence of gene flow [22,23]. Results demonstrated a mixture of both cytotypes and phenotypes within hybrid populations, suggesting a long history of gene flow. In addition, genetic marker data indicated that gene flow is bidirectional, from upland to lowland and from lowland to upland [23]. A quantitative understanding of whole switchgrass chromosomes will help in distinguishing hybrid genotypes and aid in tracking genome sources during directed breeding programs. The use of FISH and GISH can identify translocations and/or introgression of new chromosome sources $[25,65]$, but can also be used to identify ancestral genomes that contribute to the evolution of polyploid species such as switchgrass $[27,66]$.

The development of a species karyotype, with unambiguous identification of individual chromosomes, is also critical for the integration of genetic and physical map data. Genetic maps of switchgrass have been constructed using SSR and STS markers [15]. Hybridization of these genetically mapped markers to switchgrass chromosomes would lead to definitive assignment of linkage groups. Fluorescence detection of single- and low-copy sequences through the use of BAC clones as probes has proven highly successful in many species, including rice [67], maize [68], and sorghum [69]. BAC libraries for switchgrass have recently been developed and may be utilized for the integration of linkage and physical maps through BAC-FISH probing. BAC sequences would also be useful in flow sorting of chromosome fractions for physical gene mapping and construction of chromosome-specific libraries [70]. In this light, FISH technology will continue to be a valuable tool in understanding genome structure in switchgrass. 


\section{Conclusions}

The relatively small size and lack of distinguishing features among switchgrass chromosomes have likely deterred detailed karyotype analysis in the past $[11,40]$. Through the use of sophisticated molecular, cytological, and imaging techniques, we describe a quantitative karyotype for $P$. virgatum $\mathrm{L}$. that distinguishes individual chromosomes. Our data support the genetic divergence of the two subgenomes within switchgrass and provide a foundation for studying the evolution of polyploidy in this bioenergy crop. We also demonstrate karyotype differences between upland and lowland ecotypes that will aid in identifying and maintaining diverse gene pools for future breeding strategies. Additional cytogenetic analyses of switchgrass chromosomes are necessary, but data presented here provide a quantitative foundation on which genomic and genetic studies can continue to advance. This karyotype will enable aneuploid stocks as well as alien substitution and addition lines to be characterized and used by others for classical genetic analysis and introduction of new variation.

\section{Methods}

\section{Plant material and growth conditions}

Dihaploid switchgrass plants $(2 n=2 X=18)$ were identified from among the progeny of a biparental cross between two lowland tetraploid cultivars, Kanlow and Alamo, as previously described [36]. Tetraploid and octoploid cultivars used in cytological and FISH analyses were obtained either from colleagues in Lincoln, NE or from the National Genetic Resources Program (NGRP, Beltsville, MD). Cultivar accessions used in this study are described in Additional file 1 Table S1. Seeds of all ecotypes were stratified for 3 weeks at $4^{\circ} \mathrm{C}$ before germinating on wet filter paper and transplanting to the greenhouse. Plants were maintained in the greenhouse at $72^{\circ} \mathrm{F}$, under supplemental lighting (16 h day), watered as needed, and fertilized weekly with general purpose 20-20-20 fertilizer.

\section{Mitotic chromosome preparation}

Mitotic chromosome spreads were generated following a protocol by Zhang and Friebe [71] with a few modifications. Actively growing root tips were excised from greenhouse grown plants, pretreated in ice cold water for $18-24 \mathrm{~h}$, and then fixed in 3:1 ratio of $95 \%$ ethanol and glacial acetic acid at $4^{\circ} \mathrm{C}$, overnight. Root material was either used immediately for slide preparation or stored in fixative at $-20^{\circ} \mathrm{C}$ for up to several months. For slide preparation, the root tips $(0.5-1.0 \mathrm{~cm})$ were washed twice for $5 \mathrm{~min}$ in $0.01 \mathrm{M}$ citrate buffer and digested in an enzyme mixture of $50 \mathrm{mg} / \mathrm{ml}$ Onozuka R-10 cellulase and $30 \mathrm{mg} / \mathrm{ml}$ Macerozyme (Phytotechnology Labs, Shawnee Mission, KS) at $37^{\circ} \mathrm{C}$. Digestion times varied from 30 mins to $2.5 \mathrm{~h}$, depending on the thickness and degree of lignification in the root tip. Softened root tips were then washed for $5 \mathrm{~min}$ in $0.01 \mathrm{M}$ citrate buffer and transferred to a slide. Forceps and a scalpel were used to carefully excise the white tissue just behind the root cap containing actively dividing mitotic cells. All other root tissues were removed and the remaining cells were macerated in a few drops of $1 \%$ acetocarmine stain. A coverslip was placed over the stained tissue and even pressure was applied to generate mitotic chromosome spreads. Slides were viewed under phasecontrast microscopy to identify spreads optimal for use in FISH analysis.

\section{Probe DNA labeling and fluorescence in situ hybridization (FISH)}

The clone pTa71 from Triticum aestivum L. [45] as used to identify the $45 \mathrm{~S}$ rDNA (18 S-5.8 S-25 S rRNA) gene sequence. The CentC probe sequence [44] was generously provided by Dr. Zac Cande as a plasmid clone derived from PCR products of Zea mays DNA. The 5S rDNA probe for switchgrass was designed using $5 \mathrm{~S}$ rDNA sequences from maize, rice, and sorghum to identify similar sequences from a database of switchgrass 454 sequence reads from a cv. Alamo individual using BLASTN. Primers (FP 5'-AGCACGCTTACGTTC GAGTTCTGA-3'; RP 5'-AGAATGGCTAGATGCGCG GAGAAT-3') were developed from the resulting BLASTN hits with highest e-values. The PviCentC probe was amplified from switchgrass gDNA using PCR primers designed using 454 sequence reads that were identified as similar to maize $\mathrm{CentC}$ and rice CentO sequences using BLASTN [72]. Analysis of resulting BLAST hits was used to infer the PviCentC sequence from existing switchgrass raw genome sequence data. The resulting consensus sequence was used to design primers for the switchgrass-specific centromeric repeat probe (FP 5'-CATGCCCAATCCACTTCTTTAGGC-3'; RP 5'-CAACTTACGGGAAGCACAAAGTGG-3'). The resulting 143-bp PCR product was labeled with either digoxigenin-11-dUTP or biotin-16-dUTP using nick translation or PCR (Nick Translation Kit; PCR DIG Probe Synthesis Kit, Roche Applied Sciences, Indianapolis, IN), in accordance with manufacturer instructions. Hybridization and post-hybridization wash procedures were performed as previously described [73]. Chromosomes were counter-stained with 4',6-diamidino-2phenylindole (DAPI).

\section{Microscopy and image analysis}

Digital images were recorded using an Olympus BX51 epifluorescence microscope (Olympus Corporation, Center Valley, PA) with a DP70 CCD (charge coupled device) camera and suitable monochrome filter sets (Chroma 
Technology, Rockingham, VT). Images were processed using GIMP 2.6 (GNU Image Manipulation Program) for Linux. Chromosome length measurements and arm ratios were determined manually using GIMP 2.6 and confirmed with automated measurements in CHIAS IV software (Chromosome Image Analyzing System IV) [43]. Analysis of chromosome condensation patterns were performed by the CHIAS IV system using the macro programs written by Seiji Kato, Nobuko Ohmido, and Kiichi Fukui. Analysis of FISH data was conducted in GIMP 2.6 by overlaying a probe signal image on top of the corresponding DAPI stain image. Adjustments were made to the transparency of the top (FISH signal) layer to demonstrate signal and chromosome alignment.

\section{Karyotype analysis}

Karyotype analyses were performed on ten undistorted and non-overlapping chromosome spreads from the dihaploid ecotype ALB280 $(2 n=2 X=18)$. Homoeologous chromosomes from acetocarmine and/or DAPI stained spreads were paired based on total length, arm ratio, and condensation pattern. Physical length measurements were taken for each chromosome using GIMP 2.6 and CHIAS IV imaging software and by converting pixel lengths into microns. Statistical $t$ tests were conducted to confirm that homoeologous pairs were not significantly different from one another. Chromosome arms were measured from the centromere to the tip of each arm and the centricity class was determined from arm ratio ( $\mathrm{r}=$ long-arm length/short-arm length), as previously described [42]. Whenever possible, FISH signal data were used to pair homoeologous chromosomes and measurement data were averaged for each chromosome in the karyotype across all ten mitotic spreads. Chromosomes were numbered 1-9 from the longest to the shortest and paired with FISH signal data to define the karyotype $(n=9)$. In addition, condensation patterns $(\mathrm{CP})$ of all ten mitotic spreads were subjected to analysis with CHIAS IV software to aid in distinguishing chromosomes.

\section{Dihaploid lines of switchgrass}

Karyotype analysis of the base 9 chromosomes of switchgrass was significantly aided by the use of a dihaploid individual $(2 \mathrm{n}=2 \mathrm{X}=18)$ previously identified from among the progeny of a controlled cross between two tetraploid cultivars $(2 n=4 X=36)$, Kanlow and Alamo [36]. This dihaploid line (ALB280) was initially distinguished from parental lines by its reduced heterozygosity and was subsequently confirmed through estimation of $C$ values by flow cytometry. This analysis indicated that ALB280 had a $2 \mathrm{C}$ value of 1.48 , approximately half that of a tetraploid F1 reference individual (ALB881) and the Alamo male parent (ALBA4) which had $2 \mathrm{C}$ values of 2.61 and $2.75 \mathrm{pg}$, respectively [36].

\section{Additional file}

Additional file 1: Table S1. Switchgrass (Panicum virgatum L.) cultivars included in this study [74].

\section{Competing interests}

The authors declare no competing interests of any kind.

\section{Authors' contributions}

$C T, G S$, and HY conceived and designed the study. GS and $C T$ provided critical plant and molecular materials and conducted sequence analysis for the design of FISH molecular probes. HY performed the experiments and analyzed the data. HY authored the manuscript with critical help from CT and GS. All authors read and approved the final manuscript.

\section{Acknowledgments}

We thank Denise Costich and Grace Chan for critical review of the manuscript. We also thank Bernd Friebe, Rachael Wang, and Denise Costich for excellent technical advice. The U.S. Department of Agriculture, Agricultural Research Service, is an equal opportunity/affirmative action employer and all agency services are available without discrimination. Mention of commercial products and organizations in this manuscript is solely to provide specific information. It does not constitute endorsement by USDA-ARS over other products and organizations not mentioned. This work supported by the United States Department of Agriculture, Agriculture Research Service (USDA-ARS) Current Research Information System (CRIS) 5325-21000-017, USDA-ARS CRIS 5440-21000-028-00, and by a Joint USDA Department of Energy Office of Science Feedstock genomics grant DE-AI0209ER64829. The funders had no role in study design, data collection and analysis, decision to publish, or preparation of the manuscript.

\section{Author details}

${ }^{1}$ Genomics and Gene Discovery Research Unit, USDA-Agricultural Research Service, Western Regional Research Center, 800 Buchanan Street, Albany, CA 94710, USA. ²USDA Central-East Regional Biomass Center, 137 Keim Hall, East Campus, UNL, Lincoln, NE 68583, USA.

Received: 23 February 2012 Accepted: 11 July 2012

Published: 26 July 2012

\section{References}

1. Wood TE, Takebayashi N, Barker MS, Mayrose I, Greenspoon PB, Rieseberg LH: The frequency of polyploid speciation in vascular plants. Proc Natl Acad Sci 2009, 106:13875-13879.

2. Paterson AH, Bowers JE, Chapman BA: Ancient polyploidization predating divergence of the cereals, and its consequences for comparative genomics. Proc Natl Acad Sci U S A 2004, 101:9903-9908.

3. Soltis PS, Soltis DE: The role of hybridization in plant speciation. Annu Rev Plant Biol 2009, 60:561-588.

4. Doyle JJ, Doyle JL, Rauscher JT, Brown AHD: Diploid and Polyploid Reticulate Evolution Throughout the History of the Perennial Soybeans (Glycine Subgenus Glycine). New Phytol 2004, 161:121-132.

5. Aliscioni SS, Giussani LM, Zuloaga FO, Kellogg EA: A molecular phylogeny of Panicum (Poaceae: Paniceae): tests of monophyly and phylogenetic placement within the Panicoideae. Am J Bot 2003, 90:796-821.

6. Lewandowski I, Scurlock JMO, Lindvall E, Christou M: The development and current status of perennial rhizomatous grasses as energy crops in the US and Europe. Biomass Bioenergy 2003, 25:335-361.

7. McLaughlin SB, Adams Kszos L: Development of switchgrass (Panicum virgatum) as a bioenergy feedstock in the United States. Biomass Bioenergy 2005, 28:515-535.

8. Sanderson MA, Adler PR, Boateng AA, Casler MD, Sarath G: Switchgrass as a biofuels feedstock in the USA. Canadian Journal of Plant Science 2006, 86:1315-1325.

9. Church GL: Meiotic Phenomena in Certain Gramineae. II. Paniceae and Andropogoneae. Bot Gaz 1929, 88:63-84.

10. Gould FW: The Grasses of Texas. College Station: Texas A\&M University Press; 1975.

11. Church GL: Cytotaxonomic Studies in the Gramineae Spartina, Andropogon and Panicum. Am J Bot 1940, 27:263-271. 
12. Nielsen EL: Analysis of variation in Panicum virgatum L. Journal of Agriculture Research 1944, 69:327-353.

13. Brunken JN, Estes JR: Cytological and Morphological Variation in Panicum virgatum L. Southwest Nat 1975, 19:379-385.

14. Hopkins AA, Taliaferro CM, Murphy CD, Christian D: Chromosome Number and Nuclear DNA Content of Several Switchgrass Populations. Crop Sci 1996, 36:1192-1195.

15. Okada M, Lanzatella C, Saha MC, Bouton J, Wu R, Tobias CM: Complete Switchgrass Genetic Maps Reveal Subgenome Collinearity, Preferential Pairing and Multilocus Interactions. Genetics 2010, 185:745-760.

16. Vogel KP: Switchgrass. In Warm-Season (C4) Grasses. Edited by Moser L, Burson B. LE Sollenberger Madison: American Society of Agronomy; 2004:561-588.

17. Casler MD: Ecotypic Variation among Switchgrass Populations from the Northern USA. Crop Sci 2005, 45:388.

18. Costich DE, Friebe B, Sheehan MJ, Casler MD, Buckler ES: Genome-size Variation in Switchgrass (Panicum virgatum): Flow Cytometry and Cytology Reveal Rampant Aneuploidy. The Plant Genome Journal 2010, 3:130.

19. Taliaferro, Hopkins AA: Breeding characteristics and improvement potential of switchgrass. In Proceedings of the Third Liquid Fuel Conference Nashville. Edited by Cundiff JS. St Joseph, MI: TN 15-17 Sept 1996 ASAE; 1996:2-9.

20. Martínez-Reyna JM, Vogel KP: Incompatibility Systems in Switchgrass. Crop Sci 2002, 42:1800.

21. Stebbins GL: Polyploidy, Hybridization, and the Invasion of New Habitats. Annals of the Missouri Botanical Garden 1985, 72:824-832.

22. Zhang Y, Zalapa JE, Jakubowski AR, Price DL, Acharya A, Wei Y, Brummer EC, Kaeppler SM, Casler MD: Post-glacial evolution of Panicum virgatum: centers of diversity and gene pools revealed by SSR markers and cpDNA sequences. Genetica 2011, 139:933-948.

23. Zhang Y, Zalapa J, Jakubowski AR, Price DL, Acharya A, Wei Y, Brummer EC, Kaeppler SM, Casler MD: Natural Hybrids and Gene Flow between Upland and Lowland Switchgrass. Crop Sci 2011, 51:2626.

24. Young HA, Lanzatella CL, Sarath G, Tobias CM: Chloroplast genome variation in upland and lowland switchgrass. PLoS One 2011, 6:e23980.

25. D'Hont A: Unraveling the genome structure of polyploids using FISH and GISH; examples of sugarcane and banana. Cytogenet Genome Res 2005, 109:27-33.

26. Kitamura S, Tanaka A, Inoue M: Genomic relationships among Nicotiana species with different ploidy levels revealed by 5S rDNA spacer sequences and FISH/GISH. Genes Genet. Syst. 2005, 80:251-260.

27. Lim KY, Soltis DE, Soltis PS, Tate J, Matyasek R, Srubarova H, Kovarik A, Pires JC, Xiong Z, Leitch AR: Rapid chromosome evolution in recently formed polyploids in Tragopogon (Asteraceae). PLoS One 2008, 3:e3353.

28. Castilho A, Heslop-Harrison JS: Physical mapping of 5 S and 18S-25S rDNA and repetitive DNA sequences in Aegilops umbellulata. Genome 1995, 38:91-96.

29. Kato A, Lamb JC, Birchler JA: Chromosome painting using repetitive DNA sequences as probes for somatic chromosome identification in maize. Proc Natl Acad Sci U S A 2004, 101:13554-13559.

30. Shishido R, Sano Y, Fukui K: Ribosomal DNAs: an exception to the conservation of gene order in rice genomes. Mol Gen Genet 2000, 263:586-591.

31. D'Hont A, Ison D, Alix K, Roux C, Glaszmann JC: Determination of basic chromosome numbers in the genus Saccharum by physical mapping of ribosomal RNA genes. Genome 1998, 41:221-225.

32. Findley SD, Cannon S, Varala K, Du J, Ma J, Hudson ME, Birchler JA, Stacey G: A fluorescence in situ hybridization system for karyotyping soybean. Genetics 2010, 185:727-744

33. Islam-Faridi MN, Nelson CD, Kubisiak TL: Reference karyotype and cytomolecular map for loblolly pine (Pinus taeda L.). Genome 2007, 50:241-251

34. Forster BP, Heberle-Bors $E$, Kasha KJ, Touraev A: The resurgence of haploids in higher plants. Trends Plant Sci 2007, 12:368-375

35. Conger BV: Development of In Vitro Systems for Switchgrass (Panicum virgatum): Final Report for 1992 to 2002. UT-Battelle: Oak Ridge National Laboratory, UT-Battelle, US Dept. of Energy; 2002

36. Young HA, Hernlem BJ, Anderton AL, Lanzatella CL, Tobias CM: Dihaploid Stocks of Switchgrass Isolated by a Screening Approach. Bioenerg. Res. 2010, 3:305-313.
37. Maine MJD: An evaluation of the use of dihaploids and unreduced gametes in breeding for quantitative resistance to potato pathogens. J Agric Sci 1982, 99:79-83.

38. Toojinda T, Baird E, Booth A, Broers L, Hayes P, Powell W, Thomas W, Vivar H, Young G: Introgression of quantitative trait loci (QTLs) determining stripe rust resistance in barley: an example of markerassisted line development. TAG Theor Appl Genet 1998, 96:123-131.

39. Yang Z, Gilbert J, Fedak G, Somers DJ: Genetic characterization of QTL associated with resistance to Fusarium head blight in a doubled-haploid spring wheat population. Genome 2005, 48:187-196.

40. McMillan C, Weiler J: Cytogeography of Panicum virgatum in Central North America. Am J Bot 1959, 46:590-593.

41. Lu K, Kaeppler SM, Vogel KP, Arumuganathan K, Lee DJ: Nuclear DNA content and chromosome numbers in switchgrass. Great Plains Res 1998, 8:269-280

42. Levan A, Fredga K, Sandberg A: Nomenclature for Centromeric Position on Chromosomes. Hereditas 1964, 52:201-220.

43. Kato S, Fukui $\mathrm{K}$ : Condensation pattern (CP) analysis of plant chromosomes by an improved chromosome image analysing system, CHIAS III. Chromosome Res 1998, 6:473-479.

44. Ananiev EV, Phillips RL, Rines HW: Chromosome-specific molecular organization of maize (Zea mays L.) centromeric regions. Proc Natl Acad Sci U S A 1998, 95:13073-13078.

45. Gerlach WL, Bedbrook JR: Cloning and characterization of ribosomal RNA genes from wheat and barley. Nucl. Acids Res. 1979, 7:1869-1885.

46. McClintock B: A Cytological Demonstration of the Location of an Interchange Between Two Non-homologous Chromosomes of Zea mays. Proc Natl Acad Sci U S A 1930, 16:791-796.

47. Speicher MR, Carter NP: The new cytogenetics: blurring the boundaries with molecular biology. Nat Rev Genet 2005, 6:782-792.

48. Harper LC, Cande WZ: Mapping a new frontier; development of integrated cytogenetic maps in plants. Funct. Integr. Genomics 2000, 1:89-98.

49. Hamoud M, Haroun S, MacLeod R, Richards A: Cytological relationships of selected species of Panicum L. Biol Plant 1994, 36:37-45.

50. Fukui K, lijima K: Somatic chromosome map of rice by imaging methods. Theoret. Appl. Genetics 1991, 81.

51. Fukui K, Nakayama S, Ohmido N, Yoshiaki H, Yamabe M: Quantitative karyotyping of three diploid Brassica species by imaging methods and localization of $45 \mathrm{~s}$ rDNA loci on the identified chromosomes. TAG Theor App/ Genet 1998, 96:325-330.

52. Ha S, Moore P, Heinz D, Kato S, Ohmido N, Fukui K: Quantitative chromosome map of the polyploid Saccharum spontaneum by multicolor fluorescence in situ hybridization and imaging methods. Plant Mol Biol 1999, 39:1165-1173.

53. Kato S, Ohmido N, Fukui K: Development of a quantitative pachytene chromosome map in Oryza sativa by imaging methods. Genes Genet. Syst 2003, 78:155-161.

54. Langkjaer RB, Cliften PF, Johnston M, Piskur J: Yeast genome duplication was followed by asynchronous differentiation of duplicated genes. Nature 2003, 421:848-852.

55. Pendinen G, Gavrilenko T, Jiang J, Spooner DM: Allopolyploid speciation of the Mexican tetraploid potato species Solanum stoloniferum and $\mathrm{S}$. hjertingii revealed by genomic in situ hybridization. Genome 2008 51:714-720.

56. Renny-Byfield S, Ainouche M, Leitch IJ, Lim KY, Le Comber SC, Leitch AR: Flow cytometry and GISH reveal mixed ploidy populations and Spartina nonaploids with genomes of S. alterniflora and S. maritima origin. Ann Bot 2010, 105:527-533

57. Cuadrado A, Jouve N: Evolutionary trends of different repetitive DNA sequences during speciation in the genus secale. J Hered 2002, 93:339-345.

58. Thomas HM, Harper JA, Meredith MR, Morgan WG, Thomas ID, Timms E, King IP: Comparison of ribosomal DNA sites in Lolium species by fluorescence in situ hybridization. Chromosome Res. 1996, 4:486-490.

59. Hand ML, Cogan NOI, Stewart AV, Forster JW: Evolutionary history of tall fescue morphotypes inferred from molecular phylogenetics of the Lolium-Festuca species complex. BMC Evol Biol 2010, 10:303.

60. Chung M-C, Lee Y-I, Cheng Y-Y, Chou Y-J, Lu C-F: Chromosomal polymorphism of ribosomal genes in the genus Oryza. Theor Appl Genet 2008, 116:745-753. 
61. Casler MD, Stendal CA, Kapich L, Vogel KP: Genetic Diversity, Plant Adaptation Regions, and Gene Pools for Switchgrass. Crop Sci 2007, 47:2261.

62. Zalapa JE, Price DL, Kaeppler SM, Tobias CM, Okada M, Casler MD: Hierarchical classification of switchgrass genotypes using SSR and chloroplast sequences: ecotypes, ploidies, gene pools, and cultivars. Theor Appl Genet 2011, 122:805-817.

63. Martinez-Reyna J, Vogel K: Heterosis in Switchgrass: Spaced Plants. Crop Sci 2008, 48:1312-1320.

64. Vogel K, Mitchell R: Heterosis in Switchgrass: Biomass Yield in Swards. Crop Sci 2008, 48:2159-2164.

65. Zhao N, Xu L, Zhu B, Li M, Zhang H, Qi B, Xu C, Han F, Liu B: Chromosomal and genome-wide molecular changes associated with initial stages of allohexaploidization in wheat can be transit and incidental. Genome 2011, 54:692-699.

66. Xiong Z, Gaeta RT, Pires JC: Homoeologous shuffling and chromosome compensation maintain genome balance in resynthesized allopolyploid Brassica napus. Proc Natl Acad Sci 2011, 108:7908-7913.

67. Ohmido N, Akiyama Y, Fukui K: Physical mapping of unique nucleotide sequences on identified rice chromosomes. Plant Mol Biol 1998, 38:1043-1052.

68. Wang C-JR, Harper L, Cande WZ: High-Resolution Single-Copy Gene Fluorescence in Situ Hybridization and Its Use in the Construction of a Cytogenetic Map of Maize Chromosome 9. Plant Cell 2006: tpc.105.037838.

69. Kim J-S, Childs KL, Islam-Faridi MN, Menz MA, Klein RR, Klein PE, Price HJ, Mullet JE, Stelly DM: Integrated karyotyping of sorghum by in situ hybridization of landed BACs. Genome 2002, 45:402-412.

70. Doležel J, Kubaláková M, Cíhalíková J, Suchánková P, Simková H: Chromosome analysis and sorting using flow cytometry. Methods Mol. Biol 2011, 701:221-238.

71. Zhang P, Friebe B: FISH on Plant Chromosomes. In Fluorescence In Situ Hybridization (FISH) - Application Guide. Edited by Liehr T. Berlin, Heidelberg: Springer Berlin Heidelberg; 2009:365-394.

72. Altschul SF, Gish W, Miller W, Myers EW, Lipman DJ: Basic local alignment search tool. J Mol Biol 1990, 215:403-410.

73. Jenkins G, Hasterok R: BAC "landing" on chromosomes of Brachypodium distachyon for comparative genome alignment. Nat. Protocols 2007, 2:88-98.

74. Alderson JS, Sharp WC, Hanson AA, USDA: Grass varieties in the United States. Boca Raton: CRC Press; 1995.

doi:10.1186/1471-2229-12-117

Cite this article as: Young et al:: Karyotype variation is indicative of subgenomic and ecotypic differentiation in switchgrass. BMC Plant Biology 2012 12:117.

\section{Submit your next manuscript to BioMed Central and take full advantage of:}

- Convenient online submission

- Thorough peer review

- No space constraints or color figure charges

- Immediate publication on acceptance

- Inclusion in PubMed, CAS, Scopus and Google Scholar

- Research which is freely available for redistribution 